\title{
Low Complexity OFDM Detector for Underwater Acoustic Channels
}

\author{
Milica Stojanovic \\ Massachusetts Institute of Technology, MIT E38-376 \\ millitsa@mit.edu
}

\begin{abstract}
An adaptive algorithm is proposed for OFDM signal detection on Doppler-distorted, time-varying multipath channels. The focus of the approach is on low complexity post-FFT signal processing. The receiver performs MMSE combining of signals received across an array, using adaptive channel estimation. Nonuniform Doppler compensation across subbands is performed using a single adaptively estimated parameter representing the Doppler rate. Algorithm performance is demonstrated on experimental data, transmitted through a shallow water channel over the distance of $2.5 \mathrm{~km}$. QPSK modulation with a varying number of carriers is used in a $24 \mathrm{kHz}$ acoustic bandwidth. Excellent performance is achieved with up to 1024 carriers, yielding an overall bit rate of $30 \mathrm{kbps}$.
\end{abstract}

\section{INTRODUCTION}

Multi-carrier modulation is an attractive alternative to single-carrier broadband modulation on channels with frequency-selective distortion. When used with rectangular pulse shaping, multi-carrier modulation and detection are efficiently implemented using the Fast Fourier Transform. The IFFT/FFT modulator/demodulator pair of the so-obtained orthogonal frequency division multiplexing (OFDM) scheme enables channel equalization in the frequency domain, thus eliminating the need for potentially complex time-domain equalization of a single-carrier system. For this reason, OFDM has found application in a number of systems, including the wire-line Digital Subscriber Loops (DSL), wireless digital audio and video broadcast (DAB, DVB) systems, and wireless LAN (IEEE 802.11). It is also considered for the fourth generation cellular systems, and ultra-wideband (UWB) wireless communications in general.

While OFDM offers ease of channel equalization in the frequency domain, it is extremely sensitive to any frequency offset. Such an offset can result from a mismatch between the frequencies of the local oscillators, or from a Doppler distortion caused either by the transmitter/receiver motion or by a mismatch between their sampling rates. An OFDM system can only tolerate a frequency offset that is much smaller than the carrier spacing, and for this reason frequency synchronization is a critical part of the system. Any residual frequency offset causes loss of orthogonality between the carriers, and the resulting intercarrier interference (ICI) leads to performance degradation.

Methods for frequency synchronization in OFDM systems have been extensively studied, and numerous algorithms have been proposed based on the assumption that the Doppler shift is equal for all the subchannels (see e.g. [1]). When Doppler distortion is caused by motion, such an assumption is justified only in narrowband systems, where the signal bandwidth $B$ is much smaller than the center frequency. A Doppler distortion, described by the rate $a$, which is normally much less than 1 , causes the $k$ th carrier frequency $f_{k}$ to be observed at the receiver as $f_{k}+a f_{k}$. In a narrowband system with $K$ carriers spaced at $\Delta f, f_{k}>>B=K \Delta f$, and the Doppler shift can be approximated as equal for all the carriers. In a wideband OFDM system, however, this is not the case, and the Doppler distortion causes different carriers to experience substantially different frequency offsets. The need for nonuniform Doppler shift compensation across the carriers in an UWB systems is recognized in [2], where a ML estimator id provided for the Doppler rate, assuming that it is constant over one OFDM block. The ML solution is computationallyintensive, but its performance is close to the Cramer Rao bound. On time-varying channels, Doppler spreading occurs in addition to Doppler shifting of the signal, leading to further loss in performance (error floor) through additional ICI [3].

In this paper, we report on the design of an adaptive algorithm for OFDM signal detection in an UWB system. The work is motivated by applications to underwater acoustic communications, where a high-rate communication system is inherently wideband, because acoustic propagation occurs at low frequencies. In addition, the speed of sound underwater is much lower than that of electro-magnetic waves in air (nominally $c=1500 \mathrm{~m} / \mathrm{s}$ ), leading to severe motion-induced Doppler distortion. In a mobile system, an autonomous underwater vehicle (AUV) may move at a speed $v$ on the order of few $\mathrm{m} / \mathrm{s}$, with the resulting Doppler rate $a=v / c$ on the order of $10^{-3}$. Even in the absence of intentional motion, freely suspended transmitters and receivers are subject to drifting with waves and currents, at a speed that may be a fraction of a $\mathrm{m} / \mathrm{s}$ in calm conditions, or a few $\mathrm{m} / \mathrm{s}$ at rougher sea. As a result, Doppler shifting in a wideband acoustic system is not uniform across the signal bandwidth. An underwater acoustic channel is also characterized by severe multipath propagation that may produce a delay spread of a few $\mathrm{ms}$, but also as much as a hundred $\mathrm{ms}$, depending on the system location and channel conditions. The channel is time varying, with a Doppler spread that can be on the order of a Hz.

High-rate, bandwidth-efficient underwater acoustic communications have traditionally used single-carrier modulation that relies on the use of adaptive multichannel processing based on joint phase synchronization and decision-feedback equaliza- 
tion [4]. While this method has been successfully demonstrated in a variety of underwater channels, it requires careful tuning of the receiver parameters, such as the equalizer size and the parameters of the digital phase locked loop (PLL), for each deployment. Hence our motivation to investigate OFDM as a low-complexity, low-maintenance alternative for bandwidthefficient underwater acoustic communications.

In comparison with the wireless radio systems, OFDM has only been considered to a very limited extent for underwater acoustic systems. The existing work focuses mostly on conceptual system design, following the principles of narrowband radio systems, and the experimental results are scarce, e.g., [5]. The exception is the study [6] where a method is proposed for non-uniform Doppler compensation across the carriers. However, no experimental results are reported in this reference.

The goal of the present work is twofold: first, to develop a simple receiver algorithm that capitalizes on the ease of frequency-domain OFDM equalization, yet is capable of dealing with the time-varying Doppler distortion in an UWB system; and second, to demonstrate its applicability to an underwater acoustic channel using experimental data.

We consider a system with uniform energy allocation across subbands, as the channel is not known at the transmitter. The receiver performs MMSE combining of signals received across an array, using adaptive channel estimation and phase tracking. Since all the signal processing is performed digitally, there is no mismatch between the frequencies of local oscillators, and the Doppler shift is modeled as a consequence of motion-induced time compression/dilation. The Doppler rate is assumed to be constant over one OFDM block, but may vary from one block to another. In this manner, the possibility of a non-constant Doppler shift is taken into account, which is necessary when the speed and the direction of relative transmitter/receiver motion may change with time. Non-uniform Doppler compensation across subchannels is based on adaptive estimation and prediction of the Doppler rate. A single adaptively estimated parameter is thus used to track all the phases. Channel estimates are also updated on a block by block basis. Although smoothing in the frequency domain is known to improve the system performance [7], we focus at present on temporal filtering only.

We consider a zero-padded (ZP) OFDM system, instead of a conventional cyclic prefix (CP) one. ZP offers performance nearly equal to that available with $\mathrm{CP}$, as well as the possibility for improved equalization (but at increased complexity) [8]. Our choice of ZP is driven by the need to conserve transmission power. Since the guard interval between successive OFDM blocks must be at least equal to the multipath spread, filling it with CP may incur a significant waste of power when the multipath spread is comparable to the OFDM block duration. To enable same-size FFT demodulation in a ZP system, an overlap-add method of [8] is used if the multipath spread is not negligible with respect to the OFDM block duration. In doing so, we use both a section of the guard interval before, and one after the OFDM block, thus accounting for the fact that the multipath in an underwater acoustic channel is often non-causal. After overlap adding, the guard interval is discarded, and signal is demodulated using FFT, as in a CP system. Hence, the post FFT algorithm is equally applicable to both systems.

The paper is organized as follows. In Sec.II the system and channel model are specified. The receiver algorithm is presented in Sec.III. Sec.IV is devoted to the results of experimental signal processing. Conclusions are summarized in Sec.V.

\section{Channel and System Model}

We are looking at an OFDM system with $K$ subchannels, where the input data stream is serial-to-parallel converted into $K$ streams $d_{k}(n), k=0, \ldots K-1$, which are used to form the signals

$$
u_{k}(t)=\sum_{n} d_{k}(n) e^{j k \Delta \omega\left(t-n T^{\prime}\right)} g\left(t-n T^{\prime}\right)
$$

In this expression, $g(t)$ is a rectangular pulse in time with unit amplitude and duration $T ; T^{\prime}=T+T_{g}$, where $T_{g}$ is the guard interval which is longer than the multipath spread, and $\Delta \omega=2 \pi \Delta f$, where $\Delta f=1 / T$ is the carrier spacing. The signals $u_{k}(t)$ are added and shifted in frequency so that the modulated signal is given by

$$
s(t)=\operatorname{Re}\left\{\sum_{k=0}^{K-1} u_{k}(t) e^{j \omega_{0} t}\right\}
$$

where $f_{0}$ is the lowest carrier frequency, and $f_{k}=f_{0}+$ $k \Delta f$ denotes the $k$-th carrier frequency. The symbol rate is $R=K /\left(T+T_{g}\right)$ symbols per second (sps), and the signal bandwidth is defined as $B=K \Delta f$. The resulting bandwidth efficiency is $R / B=1 /\left(1+T_{g} / T\right) \mathrm{sps} / \mathrm{Hz}$.

The signal $s(t)$ passes through the channel whose impulse response, observed during the $n$th OFDM block interval $t \in$ $\left(n T^{\prime}, n T^{\prime}+T^{\prime}\right)$, is modeled by

$$
c(t, n)=\sum_{p} A_{p}(n) \delta\left(t+a(n) t-\tau_{p}(n)\right)
$$

The path gains, delays, and the Doppler rate are assumed to be constant over the block duration $T$, but they may change from one block to another. The Doppler rate is assumed equal for all paths. This assumption can be justified in systems where transmitter/receiver motion is the dominant source of Doppler distortion, and the angles of multipath arrivals differ little (e.g., horizontal shallow water transmission with range much greater than depth). The corresponding transfer function of the channel is given by

$$
C(f, n)=\sum_{p} A_{p}(n) e^{-j 2 \pi f \tau_{p}(n) /(1+a(n))}
$$

During the $n$-th block interval, the output of the demodulator in the $k$-th subchannel can be expressed as

$$
y_{k}(n)=\sum_{l=0}^{K-1} y_{k, l}(n)+w_{k}(n)
$$


where $w_{k}(n)$ is the additive noise and $y_{k, l}(n)$ is the system response to the signal $u_{l}(t)$. This signal is given by

$$
y_{k, l}(n)=c_{k}(n) \rho_{k, l} d_{l}(n) e^{j \theta_{l}(n)}
$$

where

$$
\begin{aligned}
c_{k}(n) & =C\left(f_{k}, n\right) \\
\theta_{l}(n) & =a(n) \omega_{l} T^{\prime} \\
\rho_{k, l} & =\operatorname{sinc}\left(\alpha_{k, l}\right) \cdot e^{j \alpha_{k, l}} \\
\alpha_{k, l} & =\frac{(l-k) \Delta \omega+a(n) \omega_{l}}{1+a(n)} \cdot \frac{T}{2}
\end{aligned}
$$

From the above expressions, it is clear that the OFDM demodulation principle requires the residual Doppler shift (one that exists prior to demodulation) to be much less than the carrier spacing, $a(n) f_{k}<<\Delta f, \forall k$. When this is the case, we have that $\rho_{k, k} \approx 1$, and the received signal can be represented as

$$
y_{k}(n)=c_{k}(n) d_{k}(n) e^{j \theta_{k}(n)}+z_{k}(n)
$$

where the first term contains the desired information on the symbol $d_{k}(n)$, and the second term represents residual ICI plus noise. When residual Doppler is small, $\left|\rho_{k, l}\right|<<\left|\rho_{k, k}\right|$, and the ICI can be treated as additional noise. Note also that the time-varying phase offset $\theta_{l}(n)$ helps to destroy the structure of ICI, making it look more noise-like. Hence, the major distortion to the signals remains in the time-varying phase offset $\theta_{k}(n)$. In the following section, we develop an algorithm whose focus is on estimating and removing this phase offset.

\section{ReCEIVER Algorithm}

In a multichannel receiver with $M$ spatially distributed elements, one FFT demodulator is associated with each input channel. When the elements are closely spaced, the motioninduced frequency offset is assumed to be equal for all the elements. Using the model (8) then yields the $M$-element received signal vector

$$
\mathbf{y}_{k}(n)=\mathbf{c}_{k}(n) d_{k}(n) e^{j \theta_{k}(n)}+\mathbf{z}_{k}(n)
$$

The noise components are assumed to be of equal variance $\sigma_{z}^{2}$ and uncorrelated. We note that the extension to $M$ phases is a straightforward one, but we focus on the single phase $\theta_{k}(n)$ for simplicity.

MMSE combining yields a data estimate

$$
\hat{d}_{k o}(n)=\gamma_{k}(n) \mathbf{c}_{k}^{\prime}(n) \mathbf{y}_{k}(n) e^{-j \theta_{k}(n)}
$$

where the prime denotes conjugate transpose, and

$$
\gamma_{k}(n)=\left(\sigma_{z}^{2}+\mathbf{c}_{k}^{\prime}(n) \mathbf{c}_{k}(n)\right)^{-1}
$$

In practice, the channel vector $\mathbf{c}_{k}(n)$ and the phase $\theta_{k}(n)$ are not known, and moreover, they are time-varying. Hence, their adaptive estimates are used to implement the combiner.

Using a phase estimate $\hat{\theta}_{k}(n)$ and a data symbol decision $\tilde{d}_{k}(n)$ instead of the true values to compute an unbiased leastsquares estimate of the channel vector results in an update equation

$$
\hat{\mathbf{c}}_{k}(n)=\lambda \hat{\mathbf{c}}_{k}(n-1)+(1-\lambda) \mathbf{y}_{k}(n) e^{-j \hat{\theta}_{k}(n)} \tilde{d}_{k}^{*}(n)
$$

where $\lambda$ is a positive constant less than 1 .

Once the channel estimate is available, it can be used to estimate the data symbol according to the MMSE combining rule (10). If the channel gain is varying slowly from one OFDM block to another, its estimate from the previous block, $\hat{\mathbf{c}}_{k}(n-1)$, can be used to estimate the current data symbol:

$$
\hat{d}_{k}(n)=\hat{\gamma}_{k}(n-1) \hat{\mathbf{c}}_{k}^{\prime}(n-1) \mathbf{y}_{k}(n) e^{-j \hat{\theta}_{k}(n)}
$$

The scalar $\hat{\gamma}_{k}(n-1)$ can be computed from (11) using the channel estimate and noise variance which can be obtained through a recursive least squares estimation. In a high SNR regime, however, the noise variance can be omitted, and normalization can be performed using the channel energy only, if normalization is desired at all. Normalization is not needed for PSK signals, only for QAM signals whose amplitude level influences the decision process.

Phase estimation is based on the model of motion-induced Doppler distortion:

$$
\theta_{k}(n)=\theta_{k}(n-1)+a(n) \omega_{k} T^{\prime}
$$

This model is the key to the phase tracking algorithm, which estimates the Doppler rate $a(n)$, and utilizes this single estimate to compute the phases for all the subbands. This is an important fact from the viewpoint of computational load when the number of OFDM carriers is large.

Let us assume that a phase estimate $\hat{\theta}_{k}(n-1)$ and a channel estimate $\hat{\mathbf{c}}_{k}(n-1)$ are available at the beginning of the $n$th detection interval. Upon observing the received signal $\mathbf{y}_{k}(n)$, we can calculate

$$
\hat{d}_{k 1}(n)=\hat{\gamma}_{k}(n-1) \hat{\mathbf{c}}_{k}^{\prime}(n-1) \mathbf{y}_{k}(n) e^{-j \hat{\theta}_{k}(n-1)}
$$

This quantity, however, may not be an accurate estimate of the data symbol $d_{k}(n)$. While the channel changes slowly, thus allowing us to use the past estimate $\hat{\mathbf{c}}_{k}(n-1)$, the same may not be true for the phase, which could have changed considerably over the block duration. Using the old estimate $\hat{\theta}_{k}(n-1)$ may thus lead to an angular offset between $\hat{d}_{k 1}(n)$ and the true value $d_{k}(n)$. In order to form an updated estimate $\hat{\theta}_{k}(n)$, which will then be used to make the actual data symbol estimate $\hat{d}_{k}(n)$, the angular offset in $\hat{d}_{k 1}(n)$ must first be measured. To do so, the data symbols $d_{k}(n)$ are needed, or at least some number of them. While some of the symbols can be the a-priori known pilot symbols, the others can be obtained by making tentative decisions.

To make tentative symbol decisions, a better estimate of the phase is needed than what is available from the previous block. To this end, the phase $\hat{\theta}_{k}(n-1)$ and the Doppler rate $\hat{a}(n-1)$ can be used to make a prediction on the phase $\hat{\theta}_{k}(n)$. The prediction, based on the model (14), is given by

$$
\breve{\theta}_{k}(n)=\hat{\theta}_{k}(n-1)+\hat{a}(n-1) \omega_{k} T^{\prime}
$$

This prediction is available at the beginning of the $n$th detection interval, and we use it to form

$$
\begin{gathered}
\hat{d}_{k 2}(n)=\hat{\gamma}_{k}(n-1) \hat{\mathbf{c}}_{k}^{\prime}(n-1) \mathbf{y}_{k}(n) e^{-j \breve{\theta}_{k}(n)} \\
=d_{k 1}(n) e^{-j \hat{a}(n-1) \omega_{k} T^{\prime}}
\end{gathered}
$$


This estimate is deemed reliable enough for making tentative decisions $\bar{d}_{k}(n)$. Specifically, we define

$$
\bar{d}_{k}(n)= \begin{cases}d_{k}(n), & k \in \tilde{K}(n) \\ \operatorname{decision}\left[\hat{d}_{k 2}(n)\right], & \text { otherwise }\end{cases}
$$

where $\tilde{K}(n)$ is the pool of pilot channels assigned for the $n$th detection interval. For example, we may use all the channels as pilots for $n=1$ (they will also be used to initialize the channel gain estimate) and reduce this number later. There can be only a few pilot channels, or there may be no pilot channels for $n>\tilde{N}$, some training period.

The data symbol $\bar{d}_{k}(n)$ is now used to measure the angular offset

$$
\Delta \hat{\theta}_{k}(n)=\left\langle\hat{d}_{k 1}(n) \bar{d}_{k}^{*}(n)\right\rangle
$$

This angle represents the phase change experienced in the $k$ th subband over the time interval $T^{\prime}$. If the phase change is caused by motion, i.e. it obeys the relationship (14), then the angles $\Delta \hat{\theta}_{k}(n)$ for all $k$ contain the information on the Doppler rate. One way to estimate $a(n)$ is to find the mean over all subchannels:

$$
\hat{a}(n)=\frac{1}{K} \sum_{k=0}^{K-1} \frac{\Delta \hat{\theta}_{k}(n)}{\omega_{k} T^{\prime}}
$$

Alternatively, the mean can be computed only over the pilot channels for $n \leq \tilde{N}$, switching to all subchannels later for better averaging as estimates get refined. Other ways of estimating the Doppler rate are also possible. It is also possible to filter the angular offset, e.g. as

$$
\Delta \hat{\theta}_{k}(n)=\lambda_{\theta} \Delta \hat{\theta}_{k}(n-1)+\left(1-\lambda_{\theta}\right)\left\langle\hat{d}_{k 1}(n) \bar{d}_{k}^{*}(n)\right\rangle
$$

where $\lambda_{\theta} \in[0,1)$.

Once the Doppler rate $\hat{a}(n)$ is available, we again invoke the model (14) to generate the final phase estimates for all the channels $k=0, \ldots K-1$ :

$$
\hat{\theta}_{k}(n)=\hat{\theta}_{k}(n-1)+\hat{a}(n) \omega_{k} T^{\prime}
$$

These phases are now used to make the data symbol estimates,

$\hat{d}_{k}(n)=\hat{\gamma}_{k}(n-1) \hat{\mathbf{c}}_{k}^{\prime}(n-1) \mathbf{y}_{k}(n) e^{-j \hat{\theta}_{k}(n)}=d_{k 1}(n) e^{-j \hat{a}(n) \omega_{k} T^{\prime}}$

The final symbol decisions $\tilde{d}_{k}(n)$ are made on these estimates. The symbols

$$
\tilde{d}_{k}(n)= \begin{cases}d_{k}(n), & k \in \tilde{K}(n) \\ \operatorname{decision}\left[\hat{d}_{k}(n)\right], & \text { otherwise }\end{cases}
$$

are then used to update the channel estimates according to the expression (12). The algorithm is initialized using $\hat{\mathbf{c}}_{k}(1)=$ $\mathbf{y}_{k}(1) d_{k}^{*}(1), \hat{a}(1)=0$, and $\hat{\theta}_{k}(1)=0$.

\section{Performance Demonstration}

The receiver algorithm described in the previous section was applied to a set of experimental data. Table I lists the details of the experiment, which was conducted by the Woods Hole Oceanographic Institution. The transmitter and the receiver

\begin{tabular}{|l|l|}
\hline location & Buzzards Bay, Massachusetts \\
\hline date & September 28,2005 \\
\hline tx location & $41^{\circ} 30.373^{\prime} \mathrm{N}, 70^{\circ} 43.9700 \mathrm{~W}$ \\
\hline rx location & $41^{\circ} 31.4712^{\prime} \mathrm{N}, 70^{\circ} 43.5673^{\prime} \mathrm{W}$ \\
\hline range & $2.5 \mathrm{~km}$ \\
\hline water depth & $12 \mathrm{~m}$ \\
\hline tx/rx depth & $6 \mathrm{~m}$ \\
\hline rx array elements & 12, vertical, $1.5 \mathrm{~m}$ span \\
\hline rx element spacing & $13.5 \mathrm{~cm}(\sim 3 \mathrm{wavelengths}$ at center frequency) \\
\hline tx bandwidth & $22 \mathrm{kHz}-46 \mathrm{kHz}$ \\
\hline \multicolumn{2}{|c}{ TABLE I } \\
\multicolumn{2}{|c|}{ EXPERIMENT DEPLOYMENT. }
\end{tabular}

were freely suspended from boats which were anchored in shallow water.

The modulation method was QPSK, and a varying number of subbands was used, ranging from 128 to 2048. The guard interval was chosen to be $T_{g}=25 \mathrm{~ms}$, a conservative choice for the present experiment geometry with very shallow water, but one that was made to accommodate other horizontal channels. Each frame contained a total of 32768 data symbols. The data symbols were divided into OFDM blocks, each block carrying $K$ data symbols.

OFDM modulation was performed by first generating the equivalent baseband signal $u(t)$, then translating the soobtained signal in frequency by $f_{0}$. The baseband signal was generated directly at the output sampling rate, $f_{s}=96$ $\mathrm{kHz}=4 B$, chosen to provide compatibility with varying number of subbands tested. OFDM modulation was thus implemented using IFFT of size $N_{s}=4 K$ for each block of $K$ input data symbols (with zeros appended to full length of $4 K$ ). This IFFT size is twice the minimum needed, but was chosen to avoid the need to upsample the baseband signal prior to frequency translation. The received signal was directly A/D converted using the same sampling frequency, and all processing was performed digitally. Table II summarizes the salient signal parameters.

One of the goals of the experimental work was to observe the system performance with varying number of subbands $K$. The choice of the best value for $K$ is driven by the tradeoff between the bandwidth efficiency and the system's ability to adaptively track the time-varying channel. Good bandwidth utilization is achieved at high $K$. However, as $K$ increases, so does the OFDM block duration $T$, and it becomes increasingly difficult for the adaptive algorithm to track the channel variation on a block-by-block basis. ${ }^{1}$ At the same time, the residual Doppler shift may not be negligible with respect to the carrier spacing, leading to ICI. ${ }^{2}$ By reducing $K$, the requirement on residual Doppler is relaxed, channel tracking becomes easier,

\footnotetext{
${ }^{1}$ This limitation in inherent to post-FFT processing. It can be overcome by processing the signal prior to FFT demodulation; however, computational complexity would then increase, and it is not clear whether there would be an advantage to using OFDM over adaptive equalization with single carrier modulation.

${ }^{2}$ Note that ICI suppression is also possible, but its computational complexity increases with $K$.
} 
FFT size is lower, but the bandwidth efficiency $R / B$ drops. Note also that the OFDM symbol duration $T$ may no longer be much greater than the multipath spread, in which case performance degradation will result unless time dispersion is taken into account, e.g. by overlap adding in a ZP system. Hence, there is an optimal number of carriers, and we can define it as the maximum for which reliable tracking is still possible, i.e. a pre-specified performance level is achieved.

The received signals are first digitally down-converted and frame-synchronized. The frame preamble is a PN sequence of length 127 , quadrature modulated at $24 \mathrm{ksps}$ using the center frequency of $34 \mathrm{kHz}$. Frame synchronization is performed by matched filtering to the preamble. Fig.1 illustrates the matched filter outputs obtained during one of the experiments. No Doppler shift was observed from the preamble in this case.
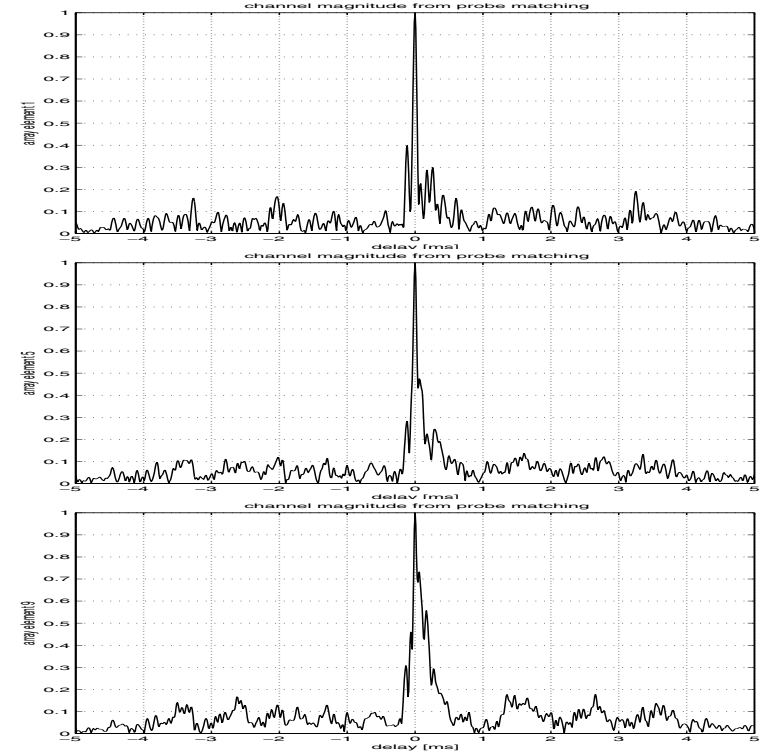

Fig. 1. Synchronization of a $K=1024$ frame: magnitude of the matched filter outputs at three of the array elements. Probe signal is a 127-element PN sequence occupying the entire bandwidth $B=24 \mathrm{kHz}$.

The results of signal processing are illustrated in Fig.2 for an OFDM signal with $K=1204$ carriers. No overlap adding is employed, as the block duration is much longer than the multipath spread. The first block is used to initialize the algorithm, and no pilot symbols are used after that. The output scatter plot shows the symbols estimates $\hat{d}_{k}(n)$. No decision errors are present in the 32 blocks of 1024 symbols each. The overall bit rate in this case is about $30 \mathrm{kbps}$.

The plot in the lower left corner shows the estimate of the Doppler rate parameter, $\hat{a}(n)$. Clearly, there is significant time-variation in the Doppler rate observed over the frame. However, the variation is slow from one block to another, which allows the receiver to track it, and the absolute level of the Doppler rate is low (not exceeding $10^{-5}$ ) such that the assumption of negligible ICI is justified. The phases corresponding to the estimated Doppler rate (only a few out of 1024 are shown) illustrate tracking of the wave-like motion. The observed Doppler shift of about $7 \mathrm{~Hz}$, and the
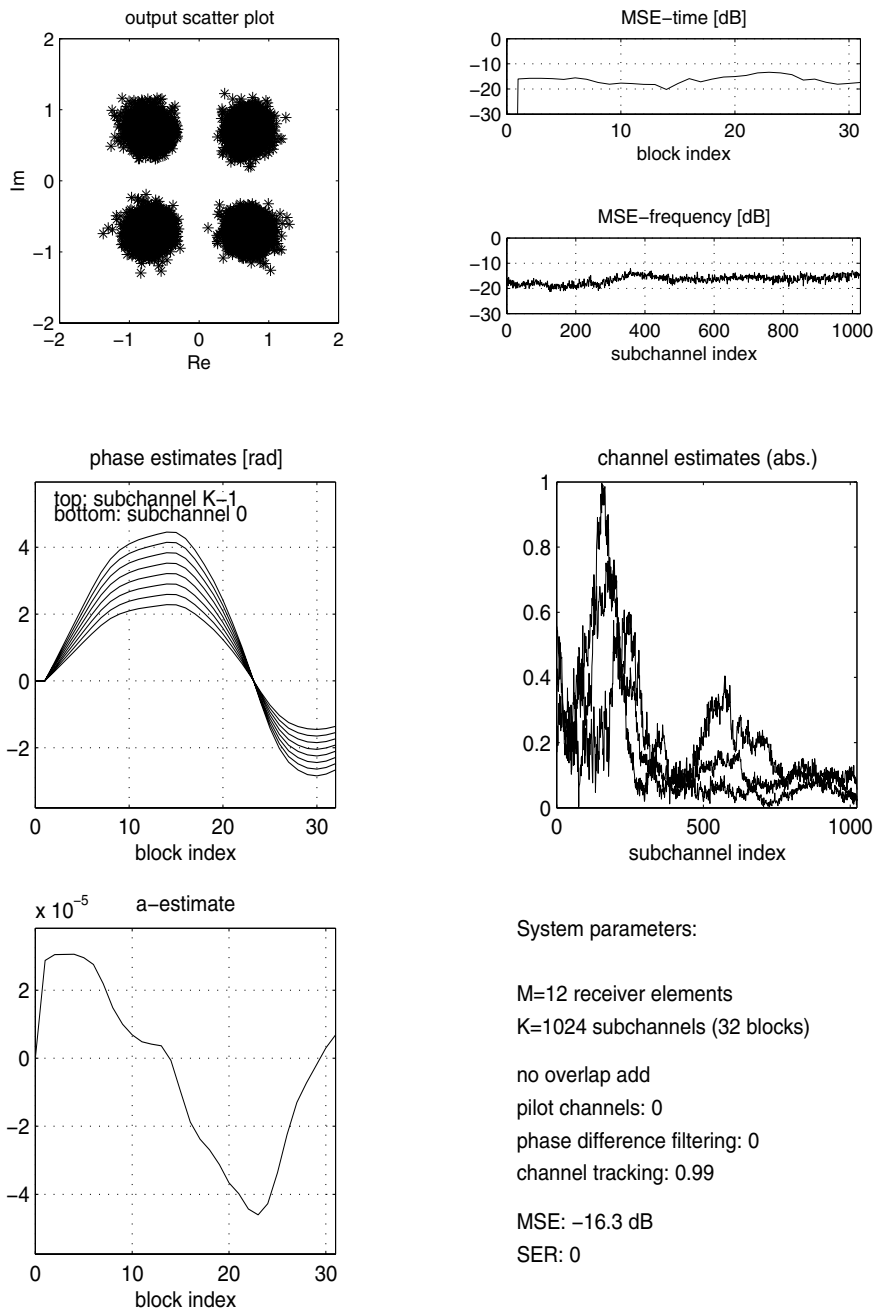

System parameters:

$\mathrm{M}=12$ receiver elements $\mathrm{K}=1024$ subchannels (32 blocks)

no overlap add

pilot channels: 0

phase difference filtering: 0

channel tracking: 0.99

MSE: $-16.3 \mathrm{~dB}$

SER: 0

Fig. 2. Signal processing results.

corresponding relative velocity of $0.25 \mathrm{~m} / \mathrm{s}$ are consistent with the experimental conditions. Judging by the performance of data detection, these phase estimates are very accurate, which serves as a justification for the model (14). We note that the phase prediction (16) proved to be a crucial step in the algorithm operation. The angular offset $\Delta \hat{\theta}(n)$ was obtained through an instantaneous measurement (19), i.e. without additional filtering.

The channel estimates represent the transfer function magnitudes for three of the receiver elements (all 12 are computed), as seen during the last OFDM block. Evidently, the channel exhibits a high degree of frequency selectivity. The channel estimates differ across the receiver array, although showing similar selectivity patterns. Time variation proves to be slow enough to enable successful tracking, but we note that adaptive channel estimation is crucial to the overall system performance. At the same time, performance sensitivity to the channel tracking constant $\lambda$ is very low.

The algorithm performance is quantified by the MSE plots shown in the upper right corner. The plot labeled "MSE-time" 


\begin{tabular}{|c|c|c|c|c|c|}
\hline \# subchannels & FFT size & carrier spacing & OFDM symbol duration & bandwidth efficiency & bit rate (QPSK) \\
\hline$K$ & $N_{s}=4 K$ & $\Delta f=B / K[\mathrm{~Hz}]$ & $T=1 / \Delta f[\mathrm{~ms}]$ & $R / B=T /(T+T g)[\mathrm{sps} / \mathrm{Hz}]$ & $\mathrm{R}[\mathrm{kbps}]$ \\
\hline 128 & $2^{9}$ & 187.5 & 5.3 & 0.17 & 0.29 \\
\hline 256 & $2^{10}$ & 93.75 & 10.6 & 0.46 & 0.63 \\
\hline 512 & $2^{11}$ & 46.87 & 21.3 & 22.1 \\
\hline 1024 & $2^{12}$ & 23.4 & 42.6 & 30.2 \\
\hline 2048 & $2^{13}$ & 11.71 & 85.3 & 0.77 \\
\hline
\end{tabular}

TABLE II

OFDM SIGNAL PARAMETERS USED FOR THE EXPERIMENT ( $B=24 k H z, T_{g}=25 \mathrm{~ms}$ ).

shows the mean squared error evaluated for each OFDM block,

$$
\operatorname{MSE}_{t}(n)=\frac{1}{K} \sum_{k=0}^{K-1}\left|d_{k}(n)-\hat{d}_{k}(n)\right|^{2}
$$

The "MSE-frequency" plot shows the mean squared error evaluated for each subband over $N$ received OFDM blocks,

$$
\operatorname{MSE}_{f}(k)=\frac{1}{N} \sum_{n=1}^{N}\left|d_{k}(n)-\hat{d}_{k}(n)\right|^{2}
$$

The system performance is uniform over time, while in frequency it is better for those bands that contain more energy. The MSE $=-16.3 \mathrm{~dB}$ indicated in the figure is the average taken over both time and frequency.

Performance was also tested with a varying number of input channels. At least three receive elements were needed to establish reliable performance, with the MSE penalty of a few $\mathrm{dB}$ with respect to the full size receiver (the exact value depends on the selection of elements and the data record used).

Hence, we conclude that the key to successful algorithm performance are the non-uniform Doppler tracking and the spatial signal combining.

The performance obtained with a varying number of carriers was excellent for all $K \leq 1024$. At $K=2048$, however, it was only possible to establish successful detection over a few blocks, but not to maintain it through block-by-block adaptation. The performance obtained with $K=128,256$ and 512 channels was similar to that obtained with $K=1024$ channels. System performance was tested over several different data records, leading to consistent observations. With $K=128$ and 256, overlap adding was necessary to ensure successful performance. It was performed using up to $4 \mathrm{~ms}$ sections of the guard interval on both sides of the useful data block. With $K \geq 512$, it offered negligible performance improvement.

\section{CONCLUSION}

Non-uniform Doppler compensation is necessary in ultrawideband OFDM systems, such as high-rate underwater acoustic systems. When Doppler distortion is caused by motion, a simple model can be used to track the phase for all carriers. Receiver design presented in this paper utilizes such a model to perform low-complexity post-FFT phase tracking and adaptive MMSE combining of signals received across an array. Receiver algorithm requires selection of a single channel-tracking constant, to which the performance is not overly sensitive. Results of experimental signal processing confirm the existence of an optimal number of carriers, for which the bandwidth efficiency is maximized, while blockby-block adaptation still provides satisfactory performance.

Future work will focus on experimental testing in varying deployment conditions, and with higher-level modulation methods. In terms of algorithm refinement, methods that exploit correlation among the subbands of the OFDM signal to perform smoothing in the frequency domain in addition to temporal filtering, are of interest. Further reduction in computational complexity should be pursued through the use of differentially coherent detection, while improvement in bandwidth efficiency should be sought through MIMO OFDM. Longer term research should address receiver feedback and the possibility to optimally allocate the signal energy across the subbands. Finally, design of algorithms for channels where different paths experience substantially different Doppler rates remains an open area.

\section{ACKNOWLEDGMENT}

I would like to thank Keenan Ball, Lee Freitag and Matt Grund of WHOI for conducting the experiment. This work was also supported in part by the NSF grant \# 0532223 and the ONR Contract N00014-05-G-0106.

\section{REFERENCES}

[1] M.Speth, S.Fechtel and H.Meyr, "Optimum receiver design for OFDM-based broadband tranmission-Part II: A case study" IEEE Trans. Commun., vol.49, No.4, pp.571-578, Apr. 2001.

[2] A-B.Salberg and A.Swami, "Doppler and frequency-offset synchronization in wideband OFDM," IEEE Trans. Wireless Commun., vol4, No.6, pp.2870-2881, Nov.2005.

[3] I.Kapoglu, Y.Li and A.Swami, "Effect of Doppler Spread in OFDMbased UWB systems," IEEE Trans. Wireless Commun., vol.4, No.6, pp.2599-2567, Sept. 2005.

[4] M.Stojanovic, J.Catipovic and J.Proakis, "Reduced-complexity multichannel processing of underwater acoustic communication signals," J. Acoust. Soc. Am., 98(2), Pt.1, pp.961-972, Aug. 1995.

[5] Frassati et al., "Experimental assessment of OFDM and DSSS modulations for use in littoral waters underwater acoustic communications," in Proc.IEEE Oceans'05 Europe Conf., June 2005.

[6] B-C.Kim and I-T.Lu, "Parameter study of OFDM Underwater Communications System,” in Proc. IEEE Oceans'00 Conf., Sept. 2000.

[7] Y.Li, L.Cimini and N.Sollenberger, "Robust channel estimation for OFDM systems with rapid dispersive fading channels," IEEE Trans. Commun., vol.46, No.7, pp.902-915, July 1998.

[8] B.Muquet, Z.Wang and G.Giannakis, "Cyclic prefix or zero padding for wireless multicarrier transmissions?," IEEE Trans. Commun., vol.50, No.12, pp.2136-2148, Dec.2002. 